3. Scriba GKE (2003) Pharmaceutical and biomedical applications of chiral capillary electrophoresis and capillary electrochromatography: an update. Electrophoresis 24(15):2409-2421

4. Bonato PS (2003) Recent advances in the determination of enantiomeric drugs and their metabolites in biological fluids by capillary electrophoresis-mediated microanalysis. Electrophoresis 24(22-23):4078-4094

5. Ghosal S, Horek J (2005) Mathematical model describing gradient focusing methods for trace analytes. Anal Chem 77(16):5380-5384

6. Urbánek M, Křivánková L, Boček P (2003) Stacking phenomena in electromigration: from basic principles to practical procedures. Electrophoresis 24(3):466-485

7. Balss KM, Vreeland WN, Phinney KW, Ross D (2004) Simultaneous concentration and separation of enantiomers with chiral temperature gradient focusing. Anal Chem 76(24):7243-7249

8. Tang GY, Yan DG, Yang C, Gong HQ, Chai JC, Lam YC (2006) Assessment of Joule heating and its effects on electroosmotic flow and electrophoretic transport of solutes in microfluidic channels. Electrophoresis 27(3):628-639

9. Probstein RF (1994) Physiochemical hydrodynamics: an introduction. Wiley, New York

10. Weast R, Astle MJ, Beyer WH (1986) CRC handbook of chemistry and physics. CRC Press, Boca Raton

\section{Temperature Gradient Generation and Control}

\author{
Barbaros Cetin ${ }^{1}$ and Dongqing $\mathrm{Li}^{2}$ \\ ${ }^{1}$ Mechanical Engineering Department, Bilkent \\ University, Ankara, Turkey \\ ${ }^{2}$ Department of Mechanical and Mechatronics \\ Engineering, Faculty of Engineering, University \\ of Waterloo, Waterloo, ON, Canada
}

\section{Synonyms}

Temperature control

\section{Definition}

In today's microfluidic technology, electrokinetic transport is used to perform many chemical and biological analyses in lab-on-a-chip devices, such as clinical diagnoses, DNA scanning, cell manipulation, cell patterning, and molecular detection. Due to the presence of an electrical potential gradient in electrokinetic transport, Joule heating is a ubiquitous phenomenon which may lead to an increase in the overall temperature and to temperature gradients in the transverse and longitudinal directions inside the channels. These generated temperature gradients and their control are crucial for the performance of the devices.

\section{Overview}

Simply, a temperature gradient can be generated by means of two temperature reservoirs at different temperatures at the inlet and the exit of a microchannel. However, in electrokinetic transport, Joule heating results in heat generation which can also result in temperature gradient generation in the longitudinal and transverse directions inside the microchannels due to the following:

- The effect of the temperature reservoirs at the inlet and the exit of the channel (thermal end effects)

- The different thermal boundary conditions at the channel wall (thermostatted and thermostatted region along the channel wall)

- The cross-sectional area change along the channel

- The flow of two parallel streams having different molar concentrations

These temperature gradients also affect the electrical field, flow field, and concentration field via temperature-dependent electrical conductivity, viscosity, and diffusivity. Large temperature gradients inside channels may cause band broadening and dispersion that leads to inefficient and low-quality separation for separation processes [1]. Effective dissipation of the heat generated by Joule heating is critical for reproducible and efficient separations in electrokinetically driven separation systems. These temperature gradients can also be useful for some applications such as isoelectric focusing (IEF) [2-4] and temperature gradient focusing (TGF) $[5,6]$. Therefore, generation and control 
of the temperature generation inside the lab-on-achip are very important to enhance and optimize the performance of the device.

Xuan and Li [7] developed an analytical model to study Joule heating effects on electrokinetic transportation in capillary electrophoresis with thermal end effects by considering the temperature-dependent fluid viscosity and electrical conductivity. They derived closed formulas for steady-state temperature field and applied electrical potential field, pressure field, velocity field, and transient concentration field which provide fundamental understanding of the effects of the temperature gradients on transport of heat, electricity, momentum, and mass species in capillary electrophoresis. They showed that Joule heating enhances the transport of samples which can provide a reduction in the analysis time for capillary electrophoretic separations. Despite the time reduction benefit, they also showed that Joule heating and thermal end effects increase the sample dispersion which leads to a lower separation efficiency. Xuan and coworkers [8,9] also studied the same effects with temperaturedependent fluid properties, both numerically [8] and experimentally [9]. They used a cagedfluorescent dye-based visualization technique to measure the EOF velocity profile and a fluorescence-based thermometry technique to measure the temperature profile inside the channel. They observed concave and convex velocity profiles due to distortion of the plug-like profile because of the induced pressure gradients resulting from the axial temperature gradient.

Nonuniform cooling across the length of the channels can be experienced by some thermostatted portion of the channel either by the following:

1. Forced convection of the channel

2. Cooling of the channel by optical infrastructure in the detection region

This nonuniform dissipation also results in temperature gradients inside the channel. Xuan and $\mathrm{Li}$ [10] studied case (1) and Sinton et al. [11] studied case (2) for a microcapillary.

Temperature gradients along the channel can also be used to focus different species inside the microchannel. IEF and TGF are the two methods that use this concept for focusing. Temperature generation along the channel is achieved both by using two reservoirs at different temperatures $[4,5]$ and by using Joule heating by means of the variable cross-sectional area along the channel $[2,3,5,6]$.

Using two parallel flow streams with different molar concentrations also results in nonuniform Joule heating and hence a temperature gradient in the transverse direction as well as the longitudinal direction. Cetin and $\mathrm{Li}$ [12] studied the effect of these two streams on the temperature field inside a microchannel for the potential use of this gradient in cell manipulation.

\section{Cross-References}

Temperature Control in Microfluidic Systems

Temperature Measurement, Methods

\section{References}

1. Li D (2004) Electrokinetics in microfluidics. Elsevier, Amsterdam

2. Pawliszyn J, Wu J (1993) Ampholyte-free isoelectric focusing of proteins in cone-shaped capillaries. J Microcolumn 5:397

3. Fang XH, Adams M, Pawliszyn J (1999) A model of thermally generated $\mathrm{pH}$ gradients in tapered capillaries. Analyst 124:335

4. Lochmuller $\mathrm{CH}$, Breiner SJ, Ronsick CS (1989) Open-channel isoelectric focusing in thermally engendered $\mathrm{pH}$ gradients. J Chromatogr 480:293

5. Ross D, Locascio LE (2002) Microfluidic temperature gradient focusing. Anal Chem 74:2556

6. Kim SM, Sommer GJ, Burns MA, Hasselbrink EF (2006) Low-power concentration and separation using temperature gradient focusing via Joule heating. Anal Chem 78:8028

7. Xuan X, Li D (2005) Analytical study of Joule heating effects on electrokinetic transportation in capillary electrophoresis. J Chromatogr A 1064:227

8. Xuan X, Sinton D, Li D (2004) Thermal end effects on electroosmotic flow in a capillary. Int $\mathrm{J}$ Heat Mass Transf 47:3145

9. Xuan X, Xu B, Sinton D, Li D (2004) Electroosmotic flow with Joule heating effects. Lab Chip 4:230

10. Xuan X, Li D (2005) Band-broadening in capillary zone electrophoresis with axial temperature gradients. Electrophoresis 26:166

11. Sinton D, Xuan X, Li D (2004) Thermally induced velocity gradients in electroosmotic microchannel 
flows: the cooling influence of optical infrastructure. Exp Fluid 37:872

12. Cetin B, Li D (2007) Modeling and Simulation of 2D, electroosmotic flow (EOF) in a slit channel with Joule heating effect. In: Proceedings of 16th national conference on thermal science, Kayseri, 30 May-2June 2007

\section{Temperature Measurement, Methods}

Vinay K. Natrajan and Kenneth T. Christensen

Department of Mechanical Science and

Engineering, University of Illinois, Urbana, IL, USA

\section{Synonyms}

Thermometry

\section{Definition}

Thermometry is the determination of a medium's temperature as inferred from properties of a probe whose characteristics are highly dependent on temperature.

\section{Overview}

There exists a growing demand for the development of advanced diagnostics for high-precision temperature measurements at the microscale. This demand is driven by the ever-increasing use of complex microfluidic devices such as micro-total analysis systems ( $\mu$-TAS) and $\mu$-heat exchangers. Lab-on-a-chip devices, which integrate multiple complex laboratory functions onto a single chip-sized substrate, often require precise temperature control to maximize the productivity of chemical operations like mixing, reactions, and separations. Such control becomes especially critical when electrokinetic pumping is used for driving flow through these devices, as the current flowing through the buffer solution can result in significant internal heat generation, a phenomenon known as Joule heating. Since most microfluidic-based MEMS devices utilize very high heat and mass transfer rates, a thorough understanding of their thermal transport characteristics is paramount for optimizing their design for increased performance and reliability.

Several methods exist for measurement of fluid and/or surface temperature at both the macro- and microscale; however, the appropriate choice of methodology is tightly coupled to the specific application under consideration. Further, the capabilities of many techniques that are commonly implemented at the macroscale are greatly challenged in microscale applications where one wishes to resolve not only the local temperature but also temperature gradients. Therefore, any technique employed in this regard must have not only satisfactory spatial resolution but also excellent temporal response since dissipation of thermal gradients at the microscale occurs over extremely short time scales owing to high heattransfer rates and large surface area-to-volume ratios. As such, obtaining accurate measurements of fluid temperature at the microscale is still a topic of intense research interest.

Until recently, temperature measurements in microfluidic systems were limited to measures of bulk fluid temperature at the inlet and outlet of microfluidic sections or simply measurement of the substrate temperature. With regard to local temperature measurements, thermocouple probes provide highly accurate measures of fluid and/or substrate temperature with excellent temporal response. However, thermocouples can often be physically intrusive and generally suffer from poor spatial resolution since most probes have a characteristic size of several microns or more. Alternatively, microfluidic devices can be fabricated with integrated microscale resistance temperature detectors (RTDs's) embedded in the substrate with spatial extents on the order of a few microns [13]. Micro-RTDs overcome the intrusiveness issues of thermocouples; however, their fabrication can be quite complex and RTDs still suffer from poor spatial resolution which limits their ability to resolve local thermal 